\title{
Active Self Supplied AC-DC Converter for Piezoelectric Energy Scavenging Systems With Supply Independent Bias
}

\author{
E. Dallago, D. Miatton, G. Venchi \\ Department of Electrical Engineering \\ University of Pavia, 27100 Pavia, Italy
}

\begin{abstract}
The paper focuses on an electronic interface which can be used into Piezoelectric Energy Scavenging Systems (PESS). These systems convert the energy of mechanical vibrations into electrical energy using a piezoelectric transducer to realize a power supply for low power electronic systems. To obtain a suitable supply source an AC-DC conversion of the output signal of these transducers is needed and, since the output power level of the energy scavenger can be very low, the conversion should be as efficient as possible.

This paper shows an active voltage doubler AC-DC converter for PESSs. A novel driving circuitry topology is presented; it has the advantage to be tolerant with respect to the process variations. The converter uses exclusively a fraction of the harvested energy to supply itself and a bias circuit has been designed to make the total current consumption supply independent. The simulation results show that the efficiency of the AC-DC converter can be as high as $94 \%$. The circuit will be diffused in $0.35 \mu \mathrm{m}$ CMOS STMicroelectronics technology.
\end{abstract}

\section{INTRODUCTION}

In recent years a lot of studies focused on energy scavenging systems which are used to harvest the normally lost environmental energy and to convert it into electrical energy. This solution can be attractive where batteries are a bottleneck for the whole system (i.e. they have a finite life time and their replacement or recharge is not feasible or too expensive). An energy scavenging system, instead, is a theoretically endless energy source. Energy scavenging systems could supersede batteries thanks to two driving forces: 1) reduction of the power consumption of the supplied electronic system, 2) optimization of the harvesting system. This last point can be further subdivided into optimization of the energy transducer and optimization of the electronic interface which has to manage and store the harvested energy.

In literature many papers can be found which describe methodologies to realize the energy-scavenger [1], [6], [8], [10]. A lot of these works are focused on the conversion of the energy associated to mechanical vibrations since they can be easily found in many environments [1], [7]. This paper describes a system based on a piezoelectric transducer since it is one of the more efficient which can be used [1]-[2]. The electrical energy at the output of this transducer is a strong and irregular function of time

\author{
V. Bottarel, G. Frattini, G. Ricotti, M. Schipani \\ STMicroelectronics \\ 20010 Cornaredo, Milan, Italy
}

[1]-[4], [9], hence, to realize a DC supply source, an AC-DC conversion is needed. The paper focuses on the optimization of the electronic interface. In particular an active voltage doubler AC-DC converter, which uses only a fraction of the harvested energy to supply its active devices, is presented.

A possible way to realize the driving circuitry of an active voltage doubler is to use comparators [3]-[4], [9] which sense the voltage drop across the switches and drive them. This approach can be tricky to be implemented, since if the value of the thresholds are different from their ideal value (e. g. because of the process spread) two phenomena can take place: 1) possible oscillation of the output of the comparators, 2) discharge of the collected charge on the piezoelectric and/or storage capacitances. Both of them could significantly worse the conversion efficiency [4]. The system is strongly dependent on the process, so the phenomena can be attenuated but not completely eliminated.

The solution proposed in this paper solves the above problems by replacing the comparators with operational amplifiers. A capacitance of $1 \mu \mathrm{F}$ is used to store the harvested energy which is partially used to supply the active circuitry of the converter so that no external power source is required, contrary to [3] and [9]. Since the voltage across the storage capacitance is variable the converter is also enriched with a circuitry which, differently from [4], makes the bias current supply independent.

A test chip will be diffused in $0.35 \mu \mathrm{m}$ CMOS STMicroelectronics technology.

\section{Piezoelectric ENERGy SCAVENGING System}

A Mechanical Aspects

The considered piezoelectric transducer is a cantilever which works in 31-mode when it is excited by the mechanical vibrations. To have a maximally efficient conversion of the mechanical vibrations into electrical energy the cantilever should be excited at its resonant frequency which can be varied adding a mass on its free end [1]. This allows the energy transducer to be tuned with the vibrations which are present in a specific environment. Some experimental measurements [1] showed that the frequency range of mechanical vibrations existing into civil environments is approximately $(60-380) \mathrm{Hz}$. 
The piezoelectric transducer can be modeled at resonance by the equivalent circuit [1]-[2] shown in Fig. $1 . \mathrm{V}_{\mathrm{PO}}$ is a sinusoidal voltage source whose frequency is equal to the transducer resonance frequency and whose amplitude is equal to the open circuit output voltage, while $C_{P}$ is the electrical capacitance of the piezoelectric cantilever.

The simulations presented in the paper are based on the model of a piezoelectric cantilever whose geometrical dimensions are $30 \times 15 \times 0.2 \mathrm{~mm} \quad(\mathrm{~L} \times \mathrm{W} \times \mathrm{H})$. The piezoelectric material used is a soft Lead Titanate Zirconate (PZT). In this case $\mathrm{C}_{\mathrm{P}}$ is equal to $19 \mathrm{nF}$, according to experimental measurements.

\section{B. Front End Circuitry}

Fig. 1 shows the active topology of the proposed AC-DC converter: switches $\mathrm{M}_{\mathrm{SW}} 1$ and $\mathrm{M}_{\mathrm{SW}} 2$ are realized with p-channel and n-channel MOSFET respectively while the driving circuitry is composed of operational amplifiers OP1 and OP2, and by the biasing circuitry. The energy stored into capacitance $C_{S}$ is also used to supply this circuitry. The DC sources indicated in Fig. 1 as $\mathrm{V}_{\mathrm{OS}}$ are the equivalent input offset voltages of the operational amplifiers. The voltage across the storage capacitance is variable; to prevent a non linear power consumption of the driving circuitry as in [4], a supply independent bias circuitry is used.

During the start-up the energy stored into $\mathrm{C}_{\mathrm{S}}$ is not enough to supply the active devices. In this case the operation of the converter is guaranteed by the body-source diode of MOSFETs $M_{S W} 1$ and $M_{S w} 2$. The proposed rectifier can be seen as a parallel of two AC-DC converters: an high efficiency active one and a lower efficiency passive one, the latter working only during start-up. The performances of the proposed solution have been simulated with a resistive load $\mathrm{R}_{\mathrm{L}}$. Since an optimum value of the output impedance exists, which maximizes the energy transfer from the PESS to the load, the efficiency was evaluated varying the load resistance between $50 \mathrm{k} \Omega$ to $1 \mathrm{M} \Omega$.

To introduce the working principle let us consider Fig. 2 which shows the case of OP1 (similar considerations apply to OP2). It is possible to apply Kirchhoff Voltage Law (KVL) to the external mesh:

$V_{I N}+V_{S D}-V_{O S}=0$

If the operational amplifier has a DC gain equal to $\mathrm{A}$ the voltage on the gate of the $\mathrm{M}_{\mathrm{SW}} 1$ is:

$V_{G}=A \cdot V_{I N}$

Equations (1) and (2) can be solved as a function of $\mathrm{V}_{\mathrm{G}}$ :

$V_{G}=A \cdot\left(V_{O S}-V_{S D}\right)$

In the ideal case the DC gain A of the operational amplifier is infinite; as a consequence the difference in equation (3) has to vanish in order to have a finite value of the gate voltage. Thus a regulation loop is obtained which maintains source to drain voltage of the MOSFET equal to offset voltage for each value of the drain current. This is obtained with a modulation of the gate voltage $\mathrm{V}_{\mathrm{G}}$.

Fig. 3 gives a graphical representation of this working principle: in the ideal case when drain current is positive the regulation loop sets the working point of the MOSFET at the intersection between its characteristics and the offset voltage. When the current decreases the loop moves the working point of the MOSFET at lower values of its source to gate voltage, until the current is equal to zero. At this point the regulation loop turns the MOSFET off; since there is no intersection between the MOSFET characteristics and the offset voltage negative values of the current are not allowed, thus the regulation loop holds the transistor off. This principle guarantees that no oscillations of the driving signal happen, solving one of the issues of [4]. Furthermore, a negative current is impeded and the discharge of the capacitances is prevented. Symmetrical considerations apply to OP2. The sign of the offset voltage is chosen to let the regulation loop work correctly. The value is not critical because it has simply to be far enough from zero so that the process mismatches will not change its sign. While it is true that an higher offset gives higher losses on the switch they are still negligible for practical offset values.

The real operational amplifier has a finite DC gain; this means that the value of the voltage across the MOSFET is slightly different from the theoretical one. Nevertheless it can be demonstrated that, with the previously described choice of the offset, this will not affect the operation of the circuit.

\section{DESIGN Of THE SUPPLy INDEPENDENT BIAS CIRCUITRY}

Since the driving circuitry should be supplied only by the harvested energy its power consumption should be as low as possible, in particular the proposed circuit has been designed to consume an average current of about $500 \mathrm{nA}$.

Furthermore a dedicated circuitry has been implemented to make this current consumption independent on the supply voltage variations.

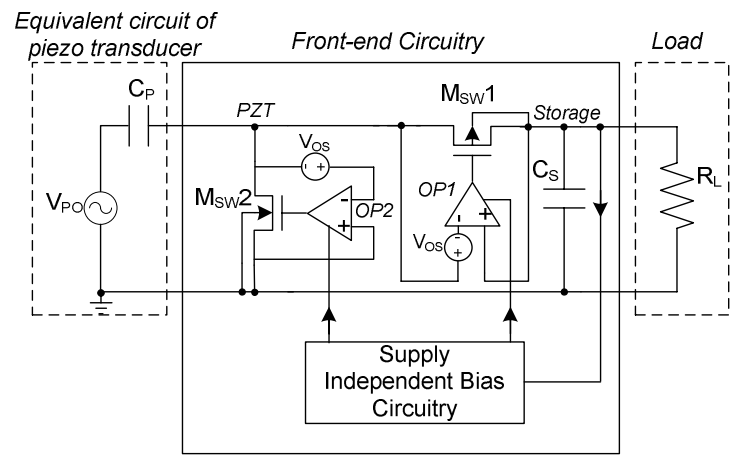

Fig. 1 Schematic of the proposed ESS. Equivalent circuit of piezoelectric transducer when it is excited at its resonant frequency, front-end circuitry and load can be identified.

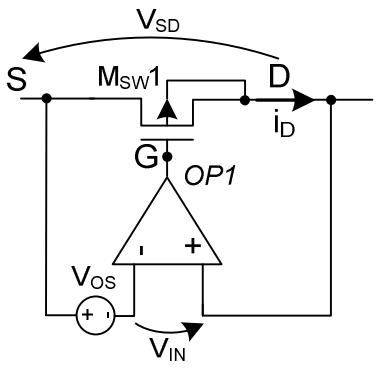

Fig. 2 Regulation loop composed by the operational amplifier OP1 and MOSFET MSW1. 


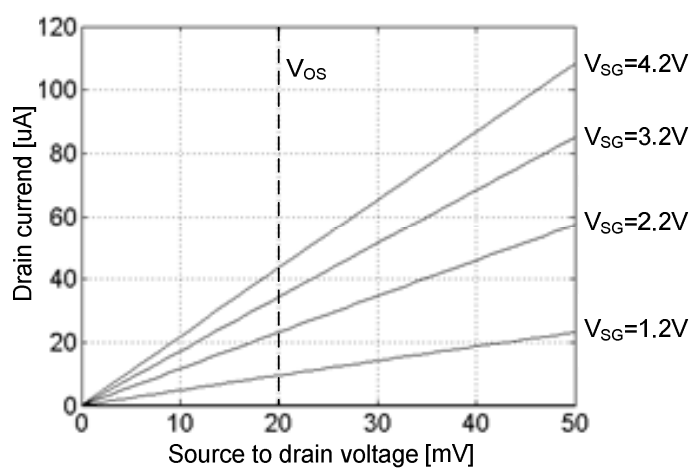

Fig. 3 Graphical solution of the regulation loop composed by the operational amplifier and MOSFET MSW1/2.

As a consequence the power consumption depends on the supply voltage in a linear way. The proposed scheme, shown in fig. 4, is modified with respect to [5] since a MOSFET connected as diode (M4) has been introduced. The system, in fact, requires supply currents in the range of tens of $\mathrm{nA}$; hence, in the scheme without M4, resistance R1 would be in the order of tens of $M \Omega$, which is too area expensive in an integrated solution. The effect of M4 is to reduce the voltage drop across $\mathrm{R} 1$ hence, for a given current I1, it has a lower value. Furthermore, a start up circuit is needed, which has been obtained with dummy MOSFETs ML1 and ML2. In particular the leakage of their body-drain diode has been exploited: its effect is to inject a current into nodes A and B so to have the start-up aid. This solution allows us to avoid additional start-up circuitry, reducing total power consumption.

Fig. 5 shows the simulated behaviour of the two bias voltages versus the supply voltage. In particular the circuitry starts to regulate when the supply voltage is higher than about $680 \mathrm{mV}$. Above this value the voltages $\mathrm{V}_{\text {BiasP }}$ and $\mathrm{V}_{\text {BiasN }}$ can be used to mirror a supply independent current.

The current consumption of the bias circuitry is about $100 \mathrm{nA}$.

\section{DESIGN OF THE OPERATIONAL AMPLIFIERS}

The schematics of the proposed operational amplifiers are shown in Fig. 6. Because of the level of their input voltages, OP1 and OP2 have to be supply compatible and ground compatible respectively.

The bias of the operational amplifiers is given by the supply independent bias circuitry. The operational amplifiers have been designed so that they are able to work with the minimum possible value of the voltage supply. In this way the active rectifier takes over the passive one as soon as a very low energy has been stored into capacitance $\mathrm{C}_{\mathrm{S}}$. A $5 \mathrm{pF}$ capacitance has been introduced to compensate the regulation loop.

Offset voltage has been obtained mismatching the aspect ratio of the input MOSFETs $\mathrm{M}_{\mathrm{A}}$ and $\mathrm{M}_{\mathrm{B}}$ : the values of the obtained offset voltages are equal to $26 \mathrm{mV}$ and $21 \mathrm{mV}$ for OP1 and OP2 respectively. Fig. 7 presents the simulation results of 500 MonteCarlo iterations, showing the possible spread of these voltages. It is possible to see that this spread is sufficiently small to have the correct sign also with process mismatches.

Aspect ratio of the MOSFETs MSW1 and MSW2 has to be chosen to avoid the loop saturation.

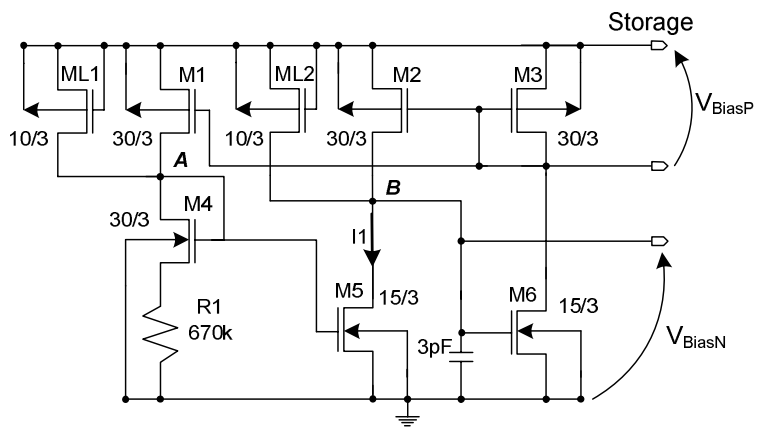

Fig. 4 Schematic of the proposed supply independent bias circuitry.

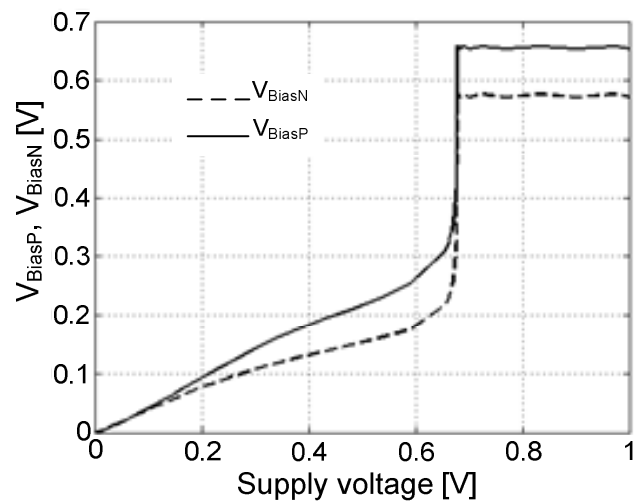

Fig. 5 Simulation results of the supply independent bias circuitry, bias voltages $\left(\mathrm{V}_{\text {BiasN }}\right.$ and $\left.\mathrm{V}_{\text {BiasP }}\right)$ versus supply voltage (in the figure supply voltage has been limited to $1 \mathrm{~V}$ for clarity. The behaviour has been verified for supply voltage up to $5 \mathrm{~V}$ )

The expression of the drain current when the MOSFET is turned on is, in a first approximation, equal to:

$i_{D}=k \frac{W}{L}\left(V_{G S}-V_{t h}\right) V_{D S}$

where $\mathrm{V}_{\text {th }}$ is the threshold voltage of the MOSFET, W/L is its aspect ratio and $\mathrm{k}$ is its characteristic constant.

The term into the parenthesis is the overdrive voltage: its value is modulated by the regulation loop which, for a each drain current, varies the gate voltage.

If the overdrive voltage is enough the source to drain voltage is equal to $\mathrm{V}_{\mathrm{OS}}$, that is:

$i_{D}=k \frac{W}{L}\left(V_{G S}-V_{t h}\right) V_{O S}$

On the other hand if, for a given current, the overdrive is not enough (which means that the op-amp is saturated), the regulation loop does not work and the source to drain voltage of $\mathrm{M}_{\mathrm{SW}} 1$ or $\mathrm{M}_{\mathrm{SW}} 2$ is not equal to offset voltage. The aspect ratio has to be designed to accommodate the maximum expected current.

Tab. 1 resumes the characteristics obtained for the amplifiers: in particular the current consumption is about $200 \mathrm{nA}$ for each amplifier. $\mathrm{V}_{\text {dd-min }}$ is the minimum supply voltage required by the operational amplifiers to work.

Fig. 8 shows the simulated efficiency of the AC-DC converter. The curves were obtained for two different 
resonant frequencies of the piezoelectric cantilever, $100 \mathrm{~Hz}$ and $200 \mathrm{~Hz}$ respectively.

The amplitude of the output voltage of the piezoelectric transducer, $\mathrm{V}_{\mathrm{PO}}$, was equal to $1.5 \mathrm{~V}$. In both cases the efficiency is higher than $80 \%$ for loads in the $(200 \mathrm{k}-1 \mathrm{M})$ Ohm range. Maximum efficiency was about $94 \%$.

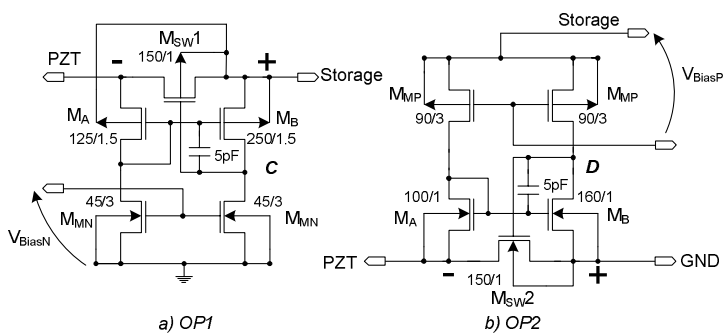

Fig. 6 Schematic of the proposed operational amplifiers.
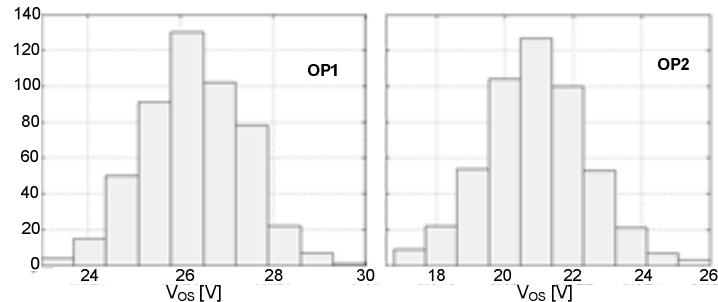

Fig. 7 MonteCarlo simulation of the offset voltages of the designed operational amplifiers.

TABLE I. CHARACTERISTICHS OF THE DESIGNED OPERATIONAL AMPLIFIERS

\begin{tabular}{|c|c|c|c|c|c|}
\hline & $\begin{array}{c}\text { GAIN-DC } \\
{[\mathbf{d B}]}\end{array}$ & $\begin{array}{c}\text { Band- } \\
\text { Width } \\
{[\mathrm{Hz}]}\end{array}$ & $\begin{array}{c}\text { Vdd- } \\
\text { min [V] }\end{array}$ & Voffset [V] & $\begin{array}{c}\text { Current } \\
\text { Consumption } \\
{[\mathbf{n A}]}\end{array}$ \\
\hline $\boldsymbol{O P 1}$ & 50.37 & 2500 & 0.73 & $26 \mathrm{e}-3$ & 200 \\
\hline $\boldsymbol{O P 2}$ & 49.78 & 2610 & 0.675 & $21 \mathrm{e}-3$ & 200 \\
\hline
\end{tabular}

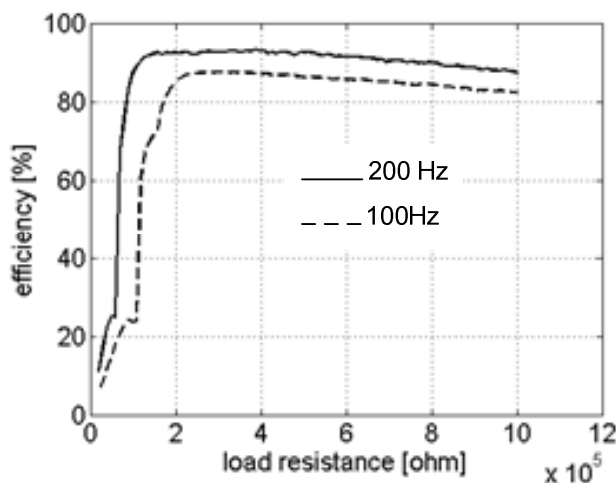

Fig. 8 Efficiency of the proposed AC-DC converter.

\section{CONCLUSIONS}

This paper presents a piezoelectric energy scavenging system where the AC-DC conversion is realized with an active voltage doubler which uses exclusively a fraction of the harvested energy to supply itself. With respect to a previous paper [4], some improvements have been introduced to obtain higher values of the efficiency, about $94 \%$, with a system which is tolerant with respect to the process variations and which is less critical to be designed. This was obtained replacing comparators with operational amplifiers which sense the voltage across the switching MOSFETs and drive their gate terminals. In this way a regulation loop exist which fixes the drop voltage across the switch at a value equal to the equivalent input offset voltage of the operational amplifier itself. This parameter can be designed with a well defined sign and with a value into a range obtained exploiting MonteCarlo simulations. Furthermore a supply independent bias circuitry has been implemented in order to make the current consumption of the driving circuitry independent on the supply voltage, contrary to [4]. Average current consumption of the whole active elements is about $500 \mathrm{nA}$.

A test chip will be diffused in CMOS $0.35 \mu \mathrm{m}$ STMicroelectronics technology.

\section{References}

[1] S. Roundy, "Energy Scavenging for Wireless Sensor Nodes with a focus on Vibration to Electricity Conversion", $\mathrm{PhD}$ Thesis, The University of California Berkeley, Spring 2003.

[2] S. Roundy et al., "Improving Power Output for Vibration-Based Energy Scavengers", Pervasive Computing January-March 2005, pp. 28-36, Published by the IEEE and IEEE ComSoc.

[3] T. T. Le, Jifeng Han, A. von Jouanne, K. Mayaram, T. S. Fiez, "Piezoelectric Micro-Power Generation Interface Circuits" IEEE Journal of Solid State Circuits, pp. 1411-1420, Vol. 41, NO. 6, June 2006.

[4] E. Dallago, G. Frattini, D. Miatton, G. Ricotti, G. Venchi, "Self-Supplied Integrable High Efficiency AC-DC Converter for Piezoelectric Energy Scavenging Systems", International Sympoium on Circuits and Systems ISCAS 2007, New Orleans LO, 27-30 May 2007, pp. 1633-1636.

[5] Z. Dong, P. E. Allen, "Low-Voltage, Supply Independent CMOS Bias Circuit", The 2002 45th Midwest Symposium on Circuits and Systems, 4-7 Aug 2002, pp. 568-570, Vol. 3.

[6] F. Peano, T. Tambosso, "Design and Optimization of a MEMS Electret-Based Capacitive Energy-Scavenger", Journal of Microelectromechanical Systems, pp.429-435Vol. 14, NO. 3, June 2005.

[7] M. Renaud, T. Sterken, P. Fiorini, R. Puers, K. Baert, C. van Hoof, "Scavenging Energy from Human Body, Design of a Piezoelectric Transducer", The 13th International Conference on Solid-State Sensors, Actuators and Mycrosystems, Seoul, Korea, June 5-9, 2005, pp. 784-787.

[8] I. Stark, "Thermal Energy Harvesting with Thermo Life ${ }^{\circledR}$, Proceedings of the International Workshop on Wearable and Implantable Body Sensor Networks, BSN 2006, pp. 19-22, IEEE Computer Society.

[9] J. Han, A. von Jouanne, T. Le, K. Mayaram, T. S. Fiez, "Novel power conditioning Circuits for Piezoelectric Micro Power Generators", APEC 2004, , February 2004, Vol. 3, pp. 1541-1546.

[10] T. Sterken, P. Fiorini, K. Baert, R. Puers, G. Borghs, "An ElectretBased Electrostatic $\mu$-generator", The 12th IEEE International Conference on Solid State Sensors, Actuators and Microsystems, Boston, June 8-12, 2003, Vol. 2, pp. 1291-1294. 